\title{
DIAGNÓSTICOS DE ENFERMERÍA COMO INSTRUMENTOS EN LA FORMACIÓN DEL ENFERMERO: UNA REVISIÓN DE LA LITERATURA
}

\author{
DIAGNÓSTICOS DE ENFERMAGEM COMO INSTRUMENTOS NA FORMAQAO DO \\ ENFERMEIRO: UMA REVISARO DE LITERATURA
}

"Carvalho da Silva, CM., "*topes de Azevedo, S., " ${ }^{* * *}$ Cavalcanti Valente, GS, ****Machado T. F. Rosas, AM., "*Marinho Chrizóstimo, M.

*Enfermeiro. Discente do Mestrado Académico em Ciencias do Cuidado em Saúde (MACCS). Escola de Enfermagem Aurora de Afonso Costa (EEAAC) Universidade Federal Fluminense (UFF). Niterói (RJ). **Mestre em Enfermagem. Professora adjunta do Depto. de Fundamentos de Enfermagem e Administracáo (MFE/ EEAAC/ UFF). Niterói (RJ). ***Doutora em Enfermagem. Professora Adjunta do Depto. de Fundamentos de Enfermagem e Administracáo (MFE/ EEAAC/ UFF). Niterói (RJ). ****Doutora em Enfermagem. Professora do Depto. Metodologia de Enfermagem. Escola de Enfermagem Anna Nery. Universidade Federal do Rio de Janeiro (UFRJ). Brasil.

Palabras clave: Diagnóstico de enfermería; Enfermería; Enseñanza

Palavras chave: Diagnóstico de Enfermagem; Enfermagem; Ensino

\section{RESUMEN}

Esta investigación presenta una revisión bibliográfica de los artículos sobre el tema "Diagnóstico de Enfermería", publicados durante los años 2000-2007, y se refiere a una tendencia que se ha afirmado en la práctica y en las revistas médicas: el estudio basado en la evidencia. Con este fin, hemos utilizado la metodología de búsqueda bibliográfica, utilizando fuentes de datos como las revistas de enfermería nacional indexadas a la base de datos LILACS (Literatura Latino-Americana y del Caribe en Ciencias de la Salud), MEDLINE (Literatura Internacional en Ciencias Salud) y SCIELO (Scientific Electronic Library Online). Se optó por utilizar el término "Diagnóstico de Enfermería", donde fueron encontrados 65 artículos. Para el análisis se puso de manifiesto la naturaleza de los artículos, la valoración de los autores, y cómo estos evalúan el diagnóstico en el hacer/educar en enfermería. Se destacaron los principales teóricos referenciados, temas y proporcionalidad de los años de la publicación en cuestión. Se puede concluir que la producción de artículos de enfermería, dentro de la temática del diagnóstico, se ha hecho incluso en pequeñas cantidades, lo que indica la necesidad de la producción y aplicación de estos productos en las esferas de la enseñanza y la práctica, teniendo en cuenta la consideración de enfermería como ciencia. 


\section{RESUMO}

Esta pesquisa apresenta uma revisáo de literatura dos artigos referentes ao tema "Diagnósticos de Enfermagem" publicados no período dos anos 2000-2007, e relaciona-os a uma tendencia, a qual cada vez tem se afirmado na prática e nas publicacóes médicas: o estudo baseado em evidencias. Para tal, utilizou-se a metodologia de pesquisa bibliográfica, utilizando-se como fontes de dados as Revistas de Enfermagem nacionais indexadas ás base de dados LILACS (Literatura LatinoAmericana e do Caribe em Ciencias da Saúde). MEDLINE (Literatura Internacional em Ciencias da Saúde) e Scielo (Scientific Electronic Library Online). Utilizando como descritor o termo "Diagnósticos de Enfermagem", foram encontrados 65 artigos. Para a análise demonstramos a natureza dos artigos, a titulacáo dos autores, e como estes avaliam a visáo diagnóstica dentro do fazer/ educar na Enfermagem, procuramos evidenciar os principais teóricos referenciados, temáticas e proporcionalidade dos anos de publicacáo em questáo. Pode-se concluir que a producáo de artigos na Enfermagem, dentro da temática diagnóstico, tem-se apresentado ainda em pequena quantidade, evidenciando a necessidade de producáo e aplicabilidade destas producóes, nos campos da docencia e prática, visto a afirmacáo da Enfermagem como ciencia.

\section{ABSTRACT}

This research presents a review of articles on the topic of "Nursing Diagnosis," published during the years 2000-2007 and refers to a trend which has increasingly been mentioned in practice and in medical journals: The study is based on evidence. To this end, we used the methodology of a bibliographic search, using data sources such as the national nursing journals indexed in the LILACS database (Latin-American and Caribbean Health Sciences Literature), MEDLINE (International Science Literature Health) SciELO (Scientific Electronic Library Online). We chose to use the term "Nursing Diagnosis," that was found in 65 articles. For the analysis it was revealed the nature of the items, the assessment of the authors and how they evaluated the diagnosis teaching of nursing. It highlighted the main theoretical references, issues of proportionality and the years of the publication in question. It can be concluded that the production of nursing articles, within the theme of the diagnosis, has been made even in small quantities, which indicates the need of production and application of these articles in the fields of teaching and practice.

\section{INTRODUCCIÓN}

La ciencia de la enfermería se basa en una amplia estructura teórica y el proceso de enfermería es un método por el cual se aplica esta estructura a la práctica. La participación del cliente en este proceso es de importancia fundamental, ofrece subvenciones para el estudio y validación de los datos, expresa sus problemas reales, tratando de lograr un intercambio de información, expectativas y experiencias. Así, se desarrolla un plan de atención para establecer mejores resultados alcanzables. (GOUVEIA; LOPES. 2004).

El proceso de enfermería consiste en una serie de pasos: la recogida de datos, diagnóstico de enfermería, planificación, ejecución y evaluación, que se centran en la individualización de la atención a través de un enfoque de la solución de los problemas que se basa en teorías y modelos conceptuales de la enfermería. Entre estos pasos, el diagnóstico de enfermería se ha puesto de relieve por el caso de un escenario dinámico, sistemático, organizado y complejo en el proceso de enfermería, es decir, no una simple lista de problemas, sino una fase que implica la evaluación crítica y toma de decisiones. (GALDEANO, 2003). 
El pensamiento crítico-reflexivo es una cadena de ideas y consecuencias, una imagen mental de algo que está presente en la realidad y que a menudo no está directamente expuesto, está impulsando la investigación y lleva a una conclusión de las metas establecidas, en donde las creencias también debe considerarse. Además de la información y las ideas relacionadas con factores relacionados, la reflexión, observación y sugerencias, son también esenciales para el pensamiento reflexivo. La reflexión es una acción para el desarrollo y enseñanza, está estrechamente relacionada con este proceso para promover la generación de los conocimientos profesionales. Es una estrategia de auto-formación, facilita al maestro cuestionar principios y problemas que afectan a su actividad. La reflexión es la reconstrucción de la experiencia docente, surge en la práctica y se convierte en un importante proceso cíclico. (BAIRRAL, 2003).

Utilizando el diagnóstico de enfermería, en una clasificación como la North American Nursing Diagnosis Association (NANDA) es posible el uso de un lenguaje común de estructura organizada. (GOUVEIA; LOPES. 2004)

El diagnóstico "es una forma de expresar las necesidades de atención identificadas en los que, como enfermeros, cuidamos. Por ejemplo, al evaluar un paciente y concluir que él tiene alto riesgo de lesiones de la piel, esa declaración - alto riesgo de lesiones de la piel, o alto riesgo para la integridad de la piel afectada - no es más que la expresión de un paciente de la condición de que, a nuestro juicio, requiere la intervención de Enfermería. Si se trata de una situación que requiere la intervención de Enfermería, es una necesidad de atención. Así podemos decir que los diagnósticos de Enfermería "expresan las necesidades de cuidados". (BRAGA, 2003)

La NANDA, contribuyendo significativamente al desarrollo y perfeccionamiento de los diagnósticos de enfermería, ha desarrollado un sistema conceptual para clasificar el diagnóstico en una taxonomía. En 1989, publicó la Taxonomía 1 y, sobre la base de diversas evaluaciones, ha publicado en 2001, la Taxonomía 2, cuya estructura fue aceptada en su conferencia bienal en 2000. (BRAGA, 2003)

La principal labor de la NANDA es la unidad de normalización de la lengua de los diagnósticos. Normalizar el lenguaje es establecer un acuerdo sobre las normas para el uso de ciertos términos. Hasta el año 2000, la clasificación de los diagnósticos de enfermería en NANDA tenía una estructura denominada Taxonomía 1, que estaba formado por nueve categorías denominadas patrones de respuestas humanas.

Esta clasificación en los patrones de respuestas humanas ha sido muy criticada. En 1994, el Comité de Taxonomía - la NANDA - se enfrentaba a graves dificultades en la clasificación de los nuevos diagnósticos que fueron aceptados, se reconoció que era posible una nueva estructura para la clasificación. Se decidió entonces verificar si surgirían conjuntos "naturales" de diagnósticos utilizando el método "Q-sort naturalístico", cuya primera ronda se concluyó en la conferencia de la NANDA 1994.

En la conferencia de 1996 fueron presentados los resultados. La estrategia ha identificado 21 categorías para clasificar los diagnósticos, y este número era muy grande para viabilizar el uso de la clasificación.

En 1998, el Comité de Taxonomía ha organizado y enviado a los directores de NANDA cuatro resultados del "Q-soif', utilizando diferentes estructuras: la primera fue el resultado del "método naturalístico" llevado a cabo entre 1994 y 1996; la segunda utilizaba la 
estructura propuesta por Jenny; la tercera utilizaba la estructura de Nursing Outcomes Classification (NOC), y la cuarta se basa en los Patrones Funcionales de Salud.

Se consideró que ninguna de estas estructuras era completamente satisfactoria, pero que la de los Patrones Funcionales de Salud es la mejor de ellas. Por lo tanto, con permiso de la autora, la NANDA ha modificado la estructura funcional de los patrones de salud y ha creado una quinta alternativa que se presentó a sus miembros en 1998.

En esta conferencia, los miembros fueron invitados a la distribución de los diagnósticos ya aceptados entre los dominios previstos por la quinta estructura. De esta etapa, resultaron 40 series de respuestas posibles de analizar. Durante la recopilación de esos datos, la Comisión tomó nota de las observaciones de la taxonomía, los problemas y las dificultades expresadas por los participantes y las sugerencias formuladas para mejorar la estructura en estudio. Con estas notas y conjuntos de respuestas, se efectuaron modificaciones adicionales a la estructura: división de un área en dos, creando un dominio que no existía y cambio del nombre de los campos a fin de reflejar mejor los diagnósticos contenidos. En la visión de la NANDA, publicada en 2001, la última estructura taxonómica no es similar a la propuesta original de Gordon, pero redujo los errores de clasificación y los valores cercanos a cero, que es una condición muy conveniente para una estructura taxonómica. (BRAGA, 2003)

La enfermería basada en la evidencia tiene sus orígenes en el movimiento de la medicina basada en evidencias y se define como "el concienzudo, explícito y juicioso uso de las mejores pruebas para tomar la decisión sobre el cuidado de los pacientes individuales", requiere habilidades que no son tradicionales en la práctica clínica, por lo tanto es necesario identificar las cuestiones clave en la toma de decisiones, buscar información científica pertinente a la cuestión y evaluar la validez de la información. La intuición, la observación no sistemática y principios fisiopatológicos se tendrán en cuenta, pero no son fuentes de pruebas con un alto grado de validez. La evidencia es "algo" que dé pruebas, y las pruebas se pueden clasificar en los niveles. (CRUZ; PIMENTA, 2005)

La evidencias deben ser buscadas para apoyar las decisiones clínicas de diagnóstico, intervenciones y resultados. El acto diagnóstico de enfermería se centra en la respuesta humana a la enfermedad, su tratamiento y los procesos de la vida. La validez de las asociaciones entre los hechos presentados por el paciente (los datos objetivos y subjetivos) y el diagnóstico es fundamental. Práctica basada en la evidencia contribuye a la precisión diagnóstica, que establece la búsqueda de los resultados de la investigación que indica que la validez. (CRUZ; PIMENTA, 2005)

Al unir la práctica diagnóstica a la enfermería evidenciado, se puede poner en práctica el servicio de atención al cliente en una forma normalizada, ya que el reconocimiento de la enfermería como ciencia comprende la formación de directrices, entre ellos el diagnóstico de enfermería. Así, se pone de manifiesto la importancia de la educación problematizadora en esta práctica, pues está experimentando que el estudiante de enfermería podrá desarrollar la competencia para diagnosticar y planificar las acciones de atención, que es esencial en la formación de enfermeras en general.

\section{METODOLOGÍA}

Este estudio consiste en una búsqueda bibliográfica, que incluye un examen completo, profundo, sistemático y crítico de las publicaciones de enfermería, y permite la creación de 
una base de conocimientos para la investigación y otras actividades especiales en el escenario de la práctica.

Según Marconi (2006), una búsqueda bibliográfica es el examen de la literatura científica, el estudio y análisis de lo que ya se ha producido en un tema en particular. A través de ella se puede tener un campo especializado del conocimiento, y por consiguiente actualización de los lectores sobre el tema en la pantalla.

La selección se hizo mediante la lectura de las publicaciones de la Enfermería brasileña publicados entre los años 2000 a 2007, que contiene artículos que abordan una visión del diagnóstico. Por la búsqueda electrónica, en revistas indexadas en la base de datos LILACS (Literatura Latino-Americana y Caribe en Ciencias de la Salud), MEDLINE (Literatura Internacional en Ciencias de la Salud) y SciELO (Scientific Electronic Library Online), se ha utilizado el descriptor "diagnóstico de enfermería" como rastreador. Se utilizaron solamente artículos de enfermería.

Se analizaron 65 artículos, entre los cuales 9 (nueve), publicados en 2000,2 (dos) en 2001 , 11 (once) en 2002, 5 (cinco) en 2003, 5 (cinco) en 2004, 14 (catorce) en 2005, 8 (ocho) en 2006 y 11 (once) artículos para el año 2007. (Figura 1)

\section{Número de publicaciones en enfermeria sobre diagnósticos de enfermeria entre los anos de 2000 y 2007}

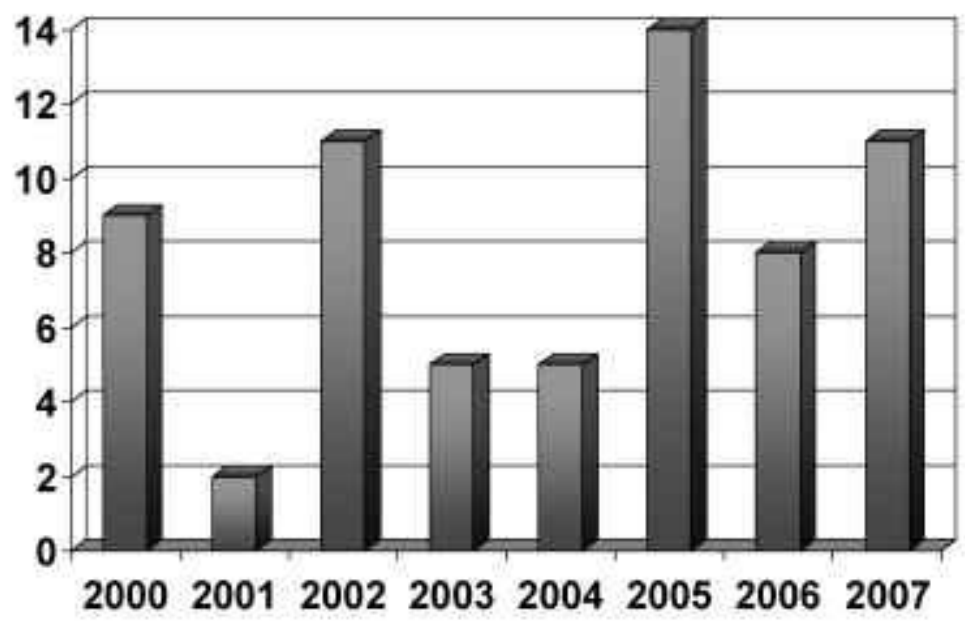

Fuente: Basis de datos LILACS, MEDLINE y SCIELO. Bùsqueda en 2008

Figura 1: Número de publicaciones en enfermeria sobre diagnósticos de enfermeria entre los años 2000 y 2007 
Los principales periódicos nacionales en que los artículos se agrupan están en el siguiente cuadro (cuadro 1):

\begin{tabular}{|c|c|c|c|c|c|c|c|c|c|}
\hline \multirow[t]{2}{*}{ PERIÓDICO } & \multicolumn{9}{|c|}{$\begin{array}{l}\text { Periodo e número de articulos publicados con el tema } \\
\text { "Diagnósticos de Enfermeria" }\end{array}$} \\
\hline & $\begin{array}{l}200 \\
0\end{array}$ & $\begin{array}{l}200 \\
1\end{array}$ & $\begin{array}{l}200 \\
2\end{array}$ & $\begin{array}{l}200 \\
3\end{array}$ & $\begin{array}{l}200 \\
4\end{array}$ & $\begin{array}{l}200 \\
5\end{array}$ & $\begin{array}{l}200 \\
6\end{array}$ & $\begin{array}{l}200 \\
7\end{array}$ & $\begin{array}{l}\text { Total } \\
\text { de } \\
\text { Artigos }\end{array}$ \\
\hline $\begin{array}{l}\text { Acta Paulista } \\
\text { de } \\
\text { Enfermagem }\end{array}$ & 3 & & 2 & 1 & 1 & 5 & 1 & 2 & 15 \\
\hline $\begin{array}{l}\text { Ciência e } \\
\text { Cuidado em } \\
\text { Saúde }\end{array}$ & & & & & 1 & & & & 1 \\
\hline $\begin{array}{l}\text { Nursing (São } \\
\text { Paulo) }\end{array}$ & & 1 & 1 & & & & & & 2 \\
\hline $\begin{array}{l}\text { Online } \\
\text { Brazilian } \\
\text { Journal of } \\
\text { Nursing }\end{array}$ & & & & & & 1 & & & 1 \\
\hline $\begin{array}{l}\text { Rev. Brasileira } \\
\text { de } \\
\text { Enfermagem }\end{array}$ & 2 & & 4 & & & 4 & & 2 & 12 \\
\hline $\begin{array}{l}\text { Rev. } \\
\text { Eletrônica de } \\
\text { Enfermagem }\end{array}$ & & & & & & & 1 & & 1 \\
\hline Rev. Enf UERJ & & & & & & & 1 & 1 & 2 \\
\hline $\begin{array}{l}\text { Rev. Esc. Enf } \\
\text { USP }\end{array}$ & & & & & & 2 & 2 & 2 & 6 \\
\hline $\begin{array}{l}\text { Rev. Gaúcha } \\
\text { de } \\
\text { Enfeermagem }\end{array}$ & & & & & & 2 & & & 2 \\
\hline $\begin{array}{l}\text { Rev. Latino- } \\
\text { americana de } \\
\text { Enfermagem }\end{array}$ & 3 & 1 & 3 & 3 & 2 & 3 & 3 & & 17 \\
\hline $\begin{array}{l}\text { Rev. Paulista } \\
\text { de } \\
\text { Enfermagem }\end{array}$ & & & 1 & 1 & & & & & 2 \\
\hline Rev. RENE & 1 & & & & 1 & & & & 2 \\
\hline $\begin{array}{l}\text { Texto e } \\
\text { Contexto em } \\
\text { Enfermagem }\end{array}$ & & & & & & & 1 & 1 & 2 \\
\hline
\end{tabular}

Cuadro 1: Periodo y número de artículos publicados con el tema "Diagnósticos de Enfermeria". Búsqueda en 2008.

\section{RESULTADOS Y ANÁLISIS}

El proceso de enfermería se caracteriza, en una de sus etapas, por la ejecución de diagnósticos de enfermería mediante el descubrimiento de los problemas del paciente y el medio ambiente que lo rodea e interactúa con él. La necesidad de la publicación de este 
tema un tanto desconocido para muchas enfermeras acostumbradas a una práctica poco direccionada se ha hecho presente en Brasil.

Según SANCHES (2004), en la década de 1980, la Ley del Ejercicio Profesional de Enfermería en Brasil tornó exclusiva a los enfermeros la prescripción de Enfermería. Como, para prescribirse debe haber un problema determinado, mucho se quiso saber sobre los diagnósticos y la clasificación en la NANDA. Aunque, de hecho, esta demanda, la autora en su estudio encontró que en el intervalo entre los años 1990 a 1999 sólo hubo 21 publicaciones en este tema.

Los 65 artículos encontrados en nuestra búsqueda, que abarca las publicaciones entre los años 2000-2007, un total de 162 autores, con un promedio de tres autores para una publicación. Sería un número importante, si no fuera por el hecho de que muchos autores participan en la autoría de más de un artículo.

Entre los autores estudiados, $9 \%$ son estudiantes becarios para pesquisas en la graduación, $11 \%$ son enfermeros generalistas, $7 \%$ enfermeros posgraduados, $32 \%$ enfermeros maestros y $41 \%$ enfermeros doctores. Cabe señalar que casi todos los autores están vinculados a la enseñanza, lo que demuestra que los profesionales del área asistencial, a pesar de tener el universo diagnóstico en su práctica, precisan contribuir con la difusión de sus conclusiones, publicando, la aplicación de la labor de las enfermeras y redescubrimiento de los parámetros de la representación de los problemas, las intervenciones y los resultados. (Figura 2)

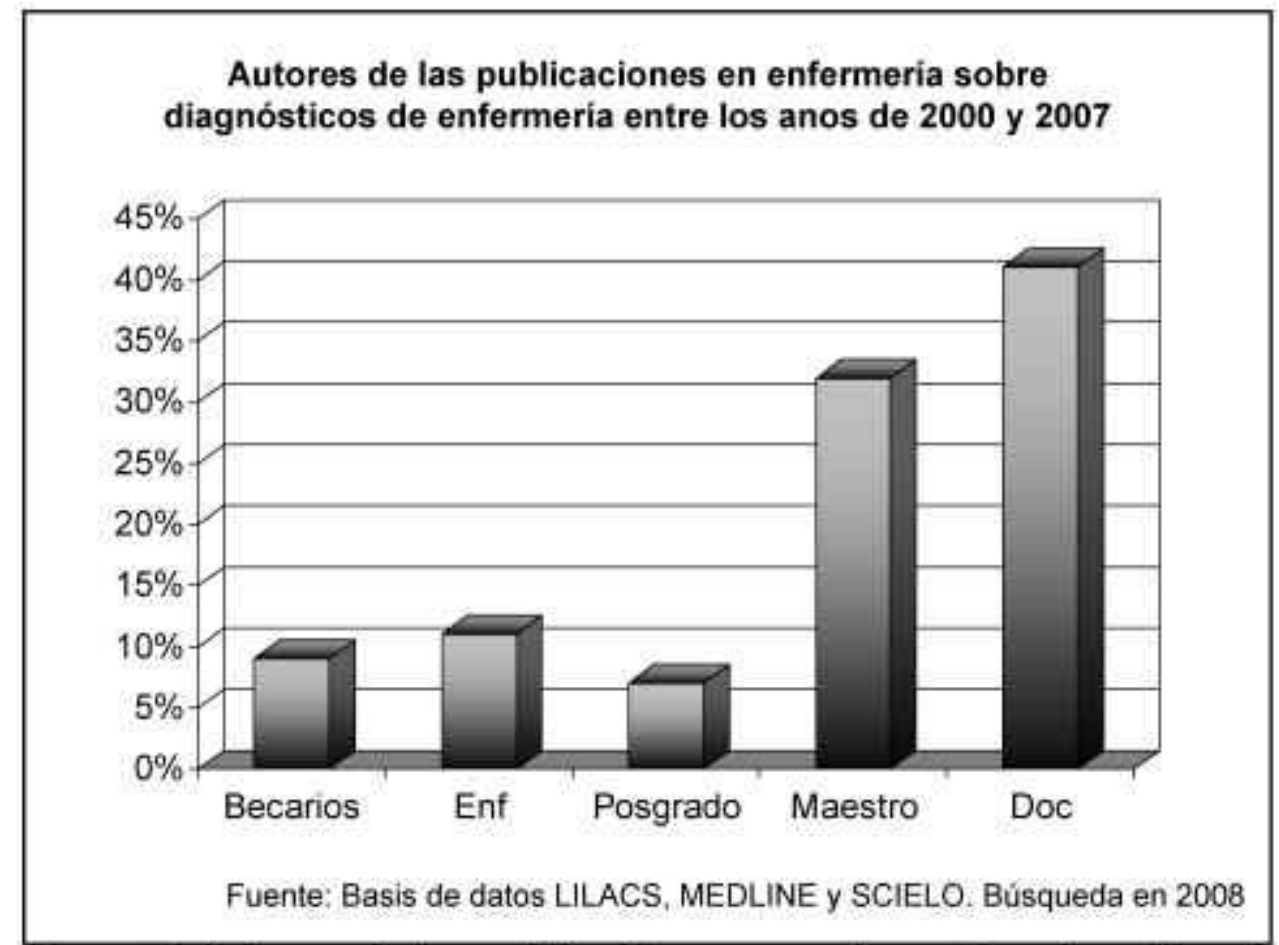

Figura 2: Autores de las publicaciones en enfermeria sobre diagnósticos de enfermeria entre los años 2000 y 2007. Búsqueda en 2008.

El enfoque utilizado en los artículos que hemos analizado, se establece de la siguiente forma: $54 \%$ de los artículos han elegido una condición o situación en que se encuentran problemas vividos como tema central del artículo, señalando los principales diagnósticos de enfermería; $10 \%$ fueron utilizados para la validación de instrumento de datos dentro de los diagnósticos de enfermería; el $12 \%$ está compuesto por estudios de las comparaciones entre grupos de ensayos donde se planteó el mismo diagnóstico de enfermería y se comparó las 
características de cada grupo; $8 \%$ retrataron las actitudes de los profesionales y los estudiantes ante el diagnóstico, sus formulaciones y definiciones; el $14 \%$ de ellas se basaron en un estudio de diagnóstico de Enfermería elegido, sus características y factores relacionados, y sólo el $2 \%$ se calcula como estudios de casos clínicos, donde se plantearon diferentes diagnósticos de Enfermería, no relacionadas con un patología específica, mas con problemas y necesidades de un cliente particular. (Figura 3 )

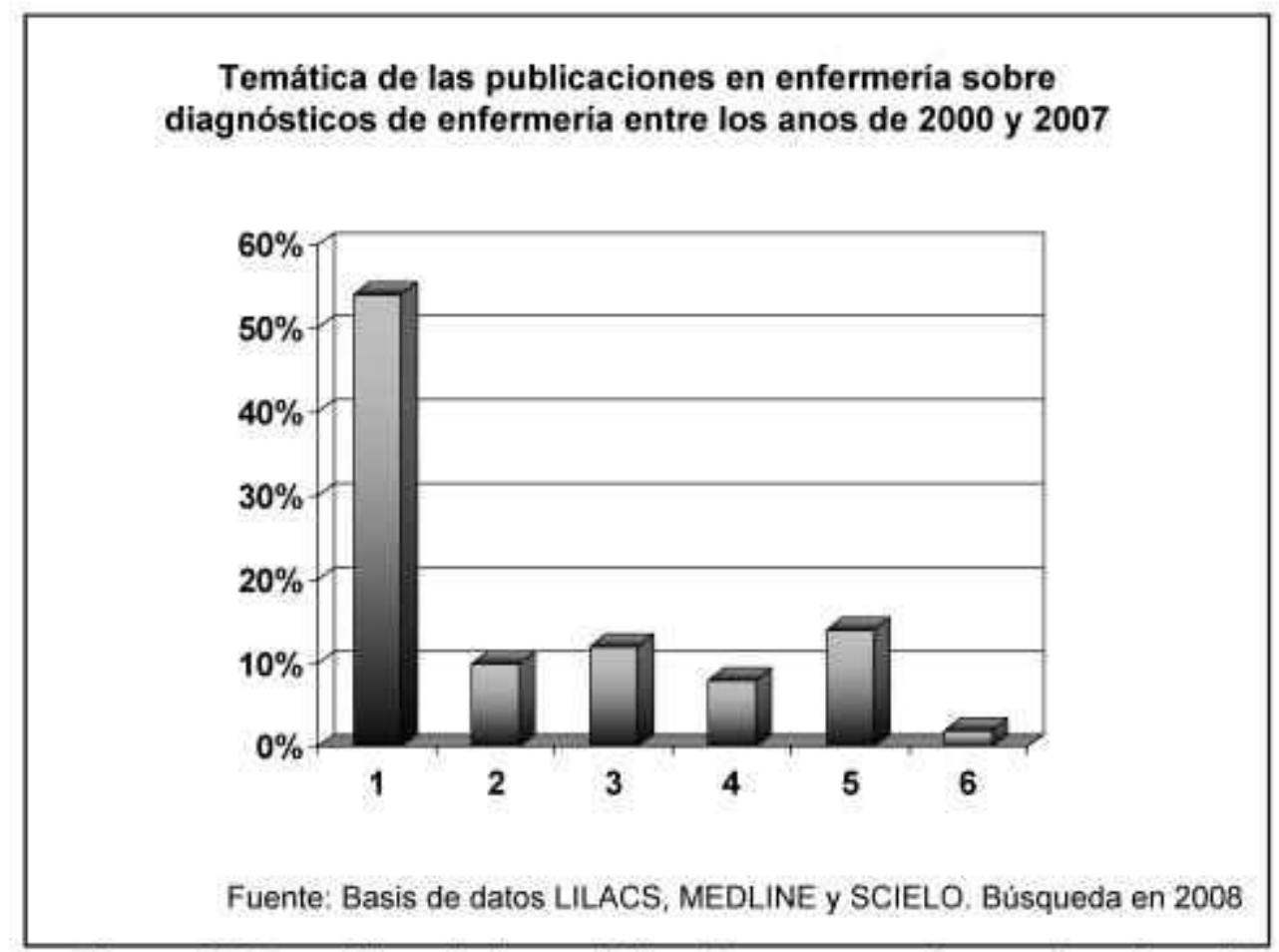

Figura 3: Temática de las publicaciones en enfermería sobre diagnósticos de enfermeria entre los años 2000 y 2007 . Búsqueda en 2008.

\author{
Representaciones: \\ 1. Condición o situación y su diagnóstico (54\%); \\ 2. Validación de instrumentos de base de datos (10\%); \\ 3. Comparación de los grupos de análisis (12\%); \\ 4. Actitud profesional a la lengua antes del diagnóstico ( $8 \%)$; \\ 5. Un estudio de diagnóstico $(14 \%$; \\ 6. Estudio de caso $(2 \%)$.
}

En cuanto a los artículos se examinaron también las pruebas que llevaron al cierre de los diagnósticos en cuestión, el referencial que llevó a las conclusiones.

Según Cruz (2005), en la práctica clínica, a menudo, la enfermera tiene que trabajar con un pequeño número de acontecimientos, interpretándolos para afirmar un diagnóstico. Los pacientes no siempre tienen todas las manifestaciones de un diagnóstico como en los libros de texto o la clasificación de los diagnósticos de enfermería. Además, muchos diagnósticos van a compartir la definición de las características, lo que dificulta el establecimiento de diagnósticos con un alto grado de precisión.

Hemos observado la necesidad de más estudios que demuestren la validez de las relaciones entre los acontecimientos y los diagnósticos, porque las pruebas deben ser buscadas en el apoyo a las decisiones clínicas de diagnóstico, las intervenciones y los resultados, ya que el 
acto en el diagnóstico de enfermería se centra en las respuestas a las enfermedades humanas y su tratamiento y los procesos de la vida. La validez de las asociaciones entre los hechos presentados por el paciente (los datos objetivos y subjetivos) y el diagnóstico es fundamental. Por lo tanto, la práctica basada en la evidencia contribuye a la precisión diagnóstica, que establece buscar los resultados de la investigación y comprobarlos por la ciencia.

Entre los principales puntos de referencia utilizados destacan las teóricas de enfermería. Una de los más citados es Wanda Horta, evidenciada en el estudio de Oliva (2005), que estudia las actitudes de los estudiantes y las enfermeras ante el diagnóstico de enfermería. La sistematización de la atención en Brasil comenzó con la investigación de la enseñanza teórica, que propone una atención organizada sobre la base de etapas (historia, diagnóstico, prescripción y de los resultados). (ZANETTI, 1992)

Farias (2000) analiza la teoría del auto-cuidado de Dorothea Orem, que incluye el autocuidado, la actividad del autocuidado. El autocuidado es la práctica de actividades iniciadas y ejecutadas por los individuos para su propio beneficio para el mantenimiento de la vida y el bienestar. La actividad de autocuiddo es una habilidad para participar en el autocuidado. La exigencia terapéutica del autocuidado constituye la totalidad de las acciones de autocuidado mediante el uso de métodos válidos y operaciones conjuntas y actividades conexas. (TORRES, DAVIN, NÓBREGA, 1999)

El autocuidado es la práctica de actividades que el individuo se inicia y se ejecuta en su propio beneficio en el mantenimiento de su vida, su salud y bienestar. Su objetivo, las acciones que, siguiendo un modelo, contribuye de manera específica en la integridad, en las funciones y en el desarrollo humano. (TORRES, DAVIN, NÓBREGA, 1999).

El Modelo Conceptual de Myra Estrin Levine aparece en los estudios de PICCOLI (2001) y Florio (2003). Esta estudiosa caracteriza el hombre como un todo dinámico, en constante interacción con el medio ambiente, más allá de este punto, este modelo se ocupa del paciente que ingresa en un establecimiento de salud necesitando asistencia, es decir, su estado de salud ha cambiado. En su modelo conceptual, Levine ha desarrollado cuatro principios de conservación: de energía, de la integridad estructural, de la integridad personal y de la integridad social del paciente. (PICOLLI, 2001)

El modelo de King (1981), estudiado por Vieira (2000), sobre mujeres con hijos prematuros ha desarrollado a través de su teoría sobre el sistema conceptual cuatro conceptos centrales que son: el medio ambiente, la salud, el ser humano y la enfermería. El sistema conceptual consta de tres sistemas de interacción: el sistema personal, que incluye la idea de que cada ser humano forma un sistema, y que a través de interacciones, esos seres humanos forman dobles, pequeños o grandes, grupos, constituyendo un nuevo sistema al que denomina sistema interpersonal. Estos grupos humanos estructuran organizaciones de acuerdo a sus necesidades e intereses, formando un nuevo sistema, el sistema social (George, 1990). Por lo tanto, el Sistema Conceptual de la teoría propuesta por King (1981) se basa en tres sistemas interactuantes y dinámicos, identificando conceptos relevantes en cada uno de elos. (VIEIRA, 2000)

Los autores han establecido definiciones prticulares sobre el diagnóstico, atribuyendo funciones a los mismos. El cuadro siguiente muestra las definiciones de estos autores, que en su totalidad otorgan ventajas al trabajo basado en el diagnóstico de las enfermeras (Cuadro 2): 


\begin{tabular}{|c|c|}
\hline \multicolumn{2}{|c|}{$\begin{array}{l}\text { Definiciones, objetivos y ventajas de Diagnósticos de Enfermeria según } \\
\text { autores estudiados }\end{array}$} \\
\hline CARVALHO (2005) & Son instrumentos que utilizan terminología propia \\
\hline CRUZ (2005) & Posibilitan correcta aplicación de intervenciones \\
\hline VIEIRA (2000) & Ayudan a la percepción de los conflictos \\
\hline FLORIO (2003) & Reber rutinas \\
\hline PICCOLI (2001) & $\begin{array}{l}\text { Muestran habilidades de enfermeria y su } \\
\text { conocimiento técnico. }\end{array}$ \\
\hline ORTIZ (2001) & Implementan educación \\
\hline COSTA (2005) & $\begin{array}{l}\text { Direccionan al acto da enfermeria, prescripción y } \\
\text { objetivos. }\end{array}$ \\
\hline SANTANA (2005) & Reconocen padrones de respuestas \\
\hline FURLAN (2003) & \\
\hline SALOMÉ (2003) & Tornan eficiente las acciones de enfermeria \\
\hline TEIXEIRA (2003) & Exigen conocimiento del enfermero \\
\hline GUIMARÄES (2000) & Llevan a la intervenciones precisas, que mejoran \\
\hline OLIVA (2005) & el estado de pacientes. \\
\hline FARIAS (2000) & Ayudan al Proceso de Enfermeria \\
\hline GUIMARÄES (2003) & Monitorizan disturbios \\
\hline CAMIA (2001) & Son, por totalidad, el plano de cuidados \\
\hline
\end{tabular}

Cuadro 2: Definiciones, objetivos y ventajas de Diagnósticos de Enfermería según autores estudiados. Búsqueda en 2008.

Incluso con todas estas ventajas, se observa que en muchos ámbitos de la práctica, el diagnóstico no se lleva a cabo, esto se refleja en la brecha de la atención, el desacuerdo entre el personal de enfermería sobre el cuidado, la demora y/o falta de curación y educación para la salud.

La educación para la salud es un conjunto de conocimientos y prácticas para la prevención de enfermedades y promoción de la salud (Costa y López, 1996). Este es un recurso mediante el cual los conocimientos científicos producidos en la salud llegan a la población por intermedio de profesionales de la salud, ayudando a la comprensión de saludenfermedad, ofreciendo mejoras para la adopción de nuevos hábitos y comportamientos.

Dentro de esta perspectiva, los diagnósticos integran el proceso desde el momento en que las intervenciones por ellos indicadas influyen en la manera de pensar y de actuar de la enfermera, que trabaja objetivando resultados para cambiar de manera consciente el modo en que el paciente ve su salud y percibe las formas de administrarla.

\section{CONCLUSIÓN}

El análisis de los estudios que han usado diagnósticos de enfermería clasificados por la NANDA nos llevó a reflexionar sobre la necesidad de los conocimientos profesionales en enfermería. Aprender a manipular la NANDA para no equivocarse en la elaboración del diagnóstico y elegir las mejores intervenciones de acuerdo a las necesidades del paciente, dependerá de cómo el proceso de enseñanza-aprendizaje se lleve a cabo en la formación de enfermeras, para que se desarrolle el nivel de competencias para la aplicación de este cuidado. 
Creemos que las enfermeras ligadas a la asistencia deben procurar actuar más intrínsecamente en el uso y la publicación de este tema, con sus conclusiones y resultados de la implementación de los cuidadosla atención, ya que este estudio encontró una tendencia en el país a la formulación de artículos con temática diagnóstica por las enfermeras relacionadas con la docencia.

La NANDA (2006) conceptualiza el diagnóstico como "un ensayo clínico sobre las respuestas del individuo, la familia o la comunidad a los procesos vitales, o para problemas de salud, actuales o potenciales, lo que proporciona la base para la selección de las prescripciones de Enfermería y para el establecimiento de resultados, de los que la enfermera es responsable", por lo tanto, es una herramienta esencial para la asistencia al desarrollo, lo que confirma la necesidad de formulación de los estudios clínicos y aplicados en hospitales, Unidades básicas de salud, en los programas y Secretarías de salud, no sólo por enfermeras comprometidas con la enseñanza.

Sanches (2005) señala, y estamos de acuerdo, en que falta una mayor divulgación y reflexión de las enfermeras en relación a la clasificación de la práctica enfermera. Formular una terminología específica depende de la realización de investigación, extensión y difusión de los resultados, y de la creatividad. La educación continua tanto para los enfermeros que trabajan en la atención como para las personas que trabajan en la educación, es otro mecanismo por el cual se pueden lograr cambios en el comportamiento.

En conclusión, nos damos cuenta de que aunque son pocas publicaciones, lo que se produce tiene buena cualidad, con informaciones notables y aprovechables sobre este tema, repletas de resultados positivos, que sin duda contribuirán en gran medida a la asistencia efectiva de enfermería basada en la evidencia.

Fue gratificante realizar este estudio dado que percibimos cuan significativa es la actuación reflexiva de la enfermera que actúa en la enseñanza de graduación en enfermería, pues de esta enseñanza no sólo deriva la realización de una asistencia humanizada, sino también el gusto por la investigación y publicación, llevando al mercado de trabajo a profesionales comprometidos con la contribución científica.

\section{BIBLIOGRAFÍA}

BAIRRAL, MA. O Crítico e o Reflexivo na Pesquisa Educacional. Uma Revisáo de Literatura. Revista Universidade Série Ciencias Humanas, v.25, 1-2, jan./dez.2003.

BRAGA, CG; CRUZ, DALM. A Taxonomia II proposta pela North American Nursing Diagnosis Association (NANDA). Rev. Latino-Am. Enfermagem., Ribeiráo Preto, v. 11, $\mathrm{n}$. 2, 2003.

CAMIA, GEK; MARIN, HF; BARBIERI, M. Diagnósticos de enfermagem em mulheres que freqüentam servigo de planejamento familiar. Rev. Latino-Am. Enfermagem., Ribeiráo Preto, v. 9, n. 2, 2001.

COSTA, JN; OLIVEIRA, MV. Fenómenos de enfermagem em portadores de lesáo medular e o desenvolvimento de úceras por pressáo. Rev. Enferm. UERJ; 13(3):367-373, set.-dez. 2005

COSTA, M.; LÓPEZ, E. Educación para la salud. Madrid: Pirámide, 1996. p.25-58.

CRUZ, DALM; PIMENTA, CAM. Prática baseada em evidencias, aplicada ao raciocínio diagnóstico. Rev. Latino-Am. Enfermagem., Ribeiráo Preto, v. 13, n. 3, 2005. 
FARIAS, MCAD; NOBREGA, MML. Diagnósticos de enfermagem numa gestante de alto risco baseados na teoria do autocuidado de Orem: estudo de caso. Rev. Latino-Am. Enfermagem., Ribeiräo Preto, v. 8, n. 6, 2000.

FLORIO, MCS; GALVAO, CM. Cirurgia ambulatorial: identificagäo dos diagnósticos de enfermagem no período perioperatório. Rev. Latino-Am. Enfermagem., Ribeiräo Preto, v. 11 , n. 5, 2003.

FURLAN, CEFB; SCOCHI, CGS; FURTADO, MCC. Percepgäo dos pais sobre a vivencia no método mäe-canguru. Revista Latino-Americana de Enfermagem (Ribeiräo Preto), Ribeiräo Preto, v. 11, n. 4, p. 444-452, 2003.

GALDEANO, LE. Diagnóstico de enfermagem de pacientes no período transoperatório de cirurgia cardíaca. Rev. Latino-Am. Enfermagem., Ribeiräo Preto, v. 11, n. 2, 2003.

GOUVEIA, HG; LOPES, MHBM. Diagnósticos de enfermagem e problemas colaborativos mais comuns na gestagäo de risco. Rev. Latino-Am. Enfermagem., Ribeiräo Preto, v. 12, n. 2, 2004.

GUIMARAES, HCQCP; BARROS, ALBL. Controlar líquidos: uma intervengäo de enfermagem para o paciente com excesso de volume de líquidos. Rev. Latino-Am. Enfermagem., Ribeiräo Preto, v. 11, n. 6, 2003.

MARCONI, MA; LAKATOS, EM. Fundamentos de metodologia científica. Säo Paulo: Atlas, 2006. $315 \mathrm{p}$.

NANDA, Diagnósticos de Enfermagem da NANDA. North American Nursing Association (org). Porto Alegre: Artes Médicas, 2006.

ORTIZ, MCA; IZANETTI, ML. Levantamento dos fatores de risco para Diabetes Mellitus tipo 2 em uma instituigäo de ensino, . Rev. Latino-Am. Enfermagem., Ribeiräo Preto, v. 9, $n$. 3, 2001.

PICCOLI, M; GALVAO, CM. Enfermagem perioperatória: identificagäo do diagnóstico de enfermagem risco para infecgäo fundamentada no modelo conceitual de levine. Rev. LatinoAm. Enfermagem., Ribeiräo Preto, v. 9, n. 4, 2001.

SANTANA, RF; Santos, I; Caldas, CP. Cuidando de idosos com demencia: um estudo a partir da pràtica ambulatorial de enfermagem. Rev. bras. enferm; 58(1):44-48, jan.-fev. 2005 TORRES, G.V.; DAVIM, R.M.B.; NÓBREGA, M.M.L. Aplicagäo do processo de enfermagem baseado na teoria de OREM estudo de caso com uma adolescente gràvida. Rev.latinoam.enfermagem, Ribeiräo Preto, v. 7, n. 2, p. 47-53, abril 1999

VIERA, CS; ROSSI, LA. Os diagnósticos de enfermagem da taxonomia da NANDA em mulheres com o filho prematuro hospitalizado e o sistema conceitual de King. Rev. LatinoAm. Enfermagem., Ribeiräo Preto, v. 8, n. 6, 2000.

ZANETTI, M.L. et al Proposta de aplicagäo do modelo conceitual de Horta utilizando a método de solugäo de problema como estrategia na assisténcia de enfermagem. In: SEMANA WANDA DE AGUIAR HORTA, 4, Säo Paulo, 1992. Programa. Säo Paulo, Escola de Enfermagem da USP, p.15-6 